University of Nebraska - Lincoln

DigitalCommons@University of Nebraska - Lincoln

Timothy J. Gay Publications

Research Papers in Physics and Astronomy

2-2019

Femtosecond-laser-induced spin-polarized electron emission from a GaAs tip

\author{
Evan M. Brunkow
}

Eric R. Jones

Herman Batelaan

Timothy J. Gay

Follow this and additional works at: https://digitalcommons.unl.edu/physicsgay

Part of the Atomic, Molecular and Optical Physics Commons, and the Other Physics Commons

This Article is brought to you for free and open access by the Research Papers in Physics and Astronomy at DigitalCommons@University of Nebraska - Lincoln. It has been accepted for inclusion in Timothy J. Gay Publications by an authorized administrator of DigitalCommons@University of Nebraska - Lincoln. 


\section{Femtosecond-laser-induced spin-polarized electron emission from a GaAs tip}

Cite as: Appl. Phys. Lett. 114, 073502 (2019); https://doi.org/10.1063/1.5070059

Submitted: 18 October 2018 . Accepted: 03 February 2019. Published Online: 20 February 2019

Evan Brunkow (D), Eric R. Jones (D), Herman Batelaan (D), and T. J. Gay (D)
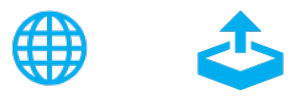

\section{Lake Shore} CRYOTRONICS

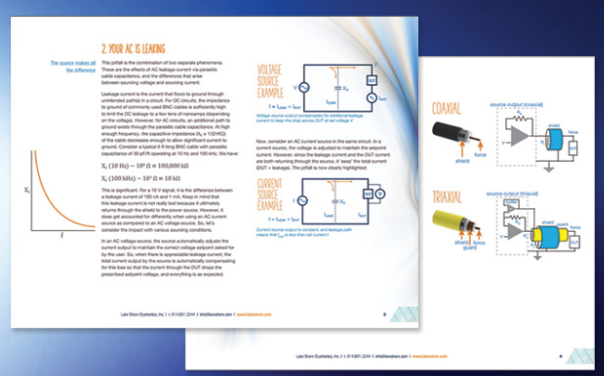

\section{5}

Electronic Measurement Pitfalls to Avoid

Get the whitepaper $\bullet$ 


\title{
Femtosecond-laser-induced spin-polarized electron emission from a GaAs tip
}

\author{
Cite as: Appl. Phys. Lett. 114, 073502 (2019); doi: 10.1063/1.5070059 \\ Submitted: 18 October 2018 • Accepted: 3 February 2019 . \\ Published Online: 20 February 2019
}

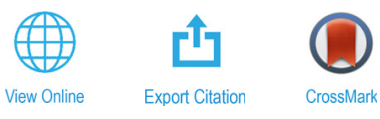

Evan Brunkow, (D) Eric R. Jones, ${ }^{\text {a) }}$ (D) Herman Batelaan, (D and T. J. Gay (D)

AFFILIATIONS

Department of Physics and Astronomy, University of Nebraska-Lincoln, Lincoln, Nebraska 68588, USA

a) eric.ryan.jones@huskers.unl.edu

\begin{abstract}
It is shown that focusing circularly polarized $800 \mathrm{~nm}$ light pulses of duration $100 \mathrm{fs}$ on the tips of $p$-GaAs crystalline shards having no negative electron affinity (NEA) activation results in electron emission that is both fast and spin-polarized. The 400 fs duration of the emission process was determined by pump/probe measurements. The three samples we investigated produced electron polarizations of 13.1(0.9)\%, 13.3(0.7)\%, and 10.4(0.2)\%. Emission currents ranged between $50 \mathrm{pA}$ and $3 \mathrm{nA}$ with a sample bias of $-100 \mathrm{~V}$ and an average laser power of $100 \mathrm{~mW}$. The electron emission exhibited linear dichroism and was obtained under moderate vacuum conditions, similar to that of metallic tips. This source of spin-polarized electron pulses is "fast" in the sense that the electron emission process is of comparable duration to the laser pulses that initiate it.
\end{abstract}

Published under license by AIP Publishing. https://doi.org/10.1063/1.5070059

Sub-picosecond, nanometer-scale, spin-polarized electron sources are currently not available. Such sources are desirable for tests of quantum degeneracy and for ultrafast electron microscopy. ${ }^{1-4}$ The first reported observation of free electron antibunching remains controversial, as the experimental apparatus could not distinguish between the effects of Coulomb pressure and degeneracy pressure.$^{5-7}$ As degeneracy pressure is polarization-dependent, while Coulomb pressure is not, a spinpolarized, sub-picosecond, nm-scale source could resolve the controversy. The best combined spatial and temporal resolutions in ultrafast electron microscopes are provided by nanotip sources triggered by femtosecond lasers, as the spatial resolution of photocathodes with planar geometry is limited by the laser focus size. ${ }^{8,9}$ Direct measurements of the electron pulse duration in ultrafast electron microscopy have shown that the electron and the illuminating laser pulse durations are of the same order. ${ }^{10}$ Implementing a spin-polarized source in an ultrafast electron microscope to study magnetic nanostructures on the fs-scale is currently under way. ${ }^{11}$

In this work, we present a fast, localized, spin-polarized source of electrons obtained from a sharp p-GaAs bulk [110] crystal shard illuminated with femtosecond laser light. The size of the emission site is approximately $1 \mu \mathrm{m}$ in scale, and the electron polarization achieved so far is $13 \%$. The electron emission was studied using methods similar to those developed to characterize pulsed emission from metallic nanotips. Such sources are currently in broad application to produce temporally short electron pulses in beams with high brightness. ${ }^{12-15}$ They are referred to as "fast," meaning that the temporal response of the emission process is comparable to that of the light pulse duration, and their spatial resolution has been shown to be determined by the size of the emitter, not the laser focus used.

Standard CW polarized electron sources use a planar GaAs photocathode that must be layered with, e.g., Cs and $\mathrm{O}_{2}$ to lower the vacuum potential below that of the conduction band. This creates a "negative electron affinity" (NEA) condition that allows electron emission by absorption of a single photon from a CW laser [Fig. 1(a)].

When circularly polarized light with an energy near the bandgap $\Delta$ of GaAs is used to excite electrons, there is an imbalance in excitation probabilities of the two excited ${ }^{2} s_{1 / 2}$ Zeeman substates [Fig. 1(c)], ${ }^{16}$ causing the emitted electrons to be spinpolarized. Such sources are used in a variety of fields, including atomic and molecular, ${ }^{17}$ high-energy, ${ }^{18}$ and condensed matter physics. $^{19}$

Alternative planar photocathodes with and without NEA have been developed to optimize the spin-polarization of the emitted electrons, to provide short pulse operation, and to enhance source brightness. NEA strained and unstrained thin 


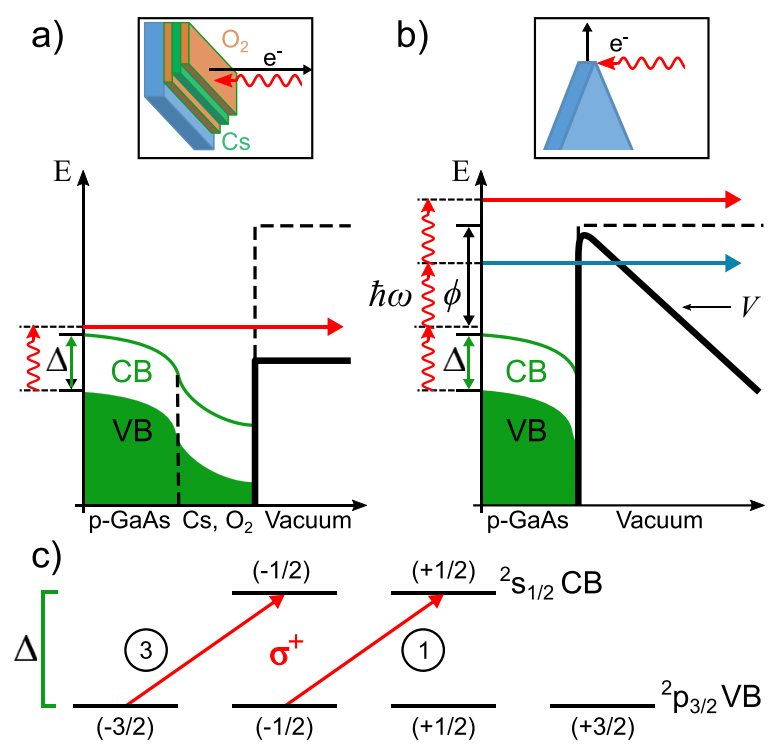

FIG. 1. GaAs energy levels for (a) NEA bulk surfaces and (b) a non-NEA shard apex. The diagrams indicate bending of both the valence band (VB) and the conduction band $(\mathrm{CB})$ at the surface due to heavy $p$-doping. (a) The vacuum energy (dashed black line) is lowered (solid black line) due to the deposition of alternating layers of $\mathrm{Cs}$ and $\mathrm{O}_{2}$ (top inset). Electron emission from the NEA surface proceeds by the absorption of a single photon with energy that exceeds the bandgap $\Delta$ of the bulk. (b) Multiphoton emission from an uncoated, non-NEA GaAs shard apex (see the text). (c) Allowed transitions at the GaAs $\Gamma$-point for absorption of righthand circularly polarized light by Zeeman $\left(m_{j}\right)$ sublevels. Selection rules $\left(\Delta m_{j}\right.$ $=+1)$ and the relative line strengths (indicated in circles) yield a nascent conductionband electron polarization of $(3-1) /(3+1)=50 \%$ for valence-conduction band resonant transitions.

photocathodes have produced 2.5 ps electron pulses. ${ }^{20}$ Here, the electron pulse duration is limited by the slow emission process of diffusion through the material. A strained, back-illuminated GaAs-GaAsP superlattice with NEA activation resulted in a 16 ps pulse duration with high brightness ${ }^{21-23}$ and was used in a spinpolarized transmission electron microscope. The source was determined to have a degeneracy 2 orders of magnitude lower than the cathode tip used to first study free electron degeneracy, ${ }^{5}$ with a source size that was limited by the diffraction limit of the laser focus. A planar GaAs photocathode with a Ag overlayer a few nm thick has functioned as a polarized electron source without NEA activation by utilizing a multiphoton electron emission process. ${ }^{24}$ Electron yields were increased by employing local field enhancement through plasmonic coupling on the surface of a $p$-doped GaAs wafer, while the spin-polarization of emitted electrons was largely maintained. Pulsed $\sim 100$ fs laser light produced a spin-polarization as high as $21 \%$, with a value of $\sim 15 \%$ for illumination at a central wavelength of $800 \mathrm{~nm}$.

Tips of magnetized iron and cobalt-coated tungsten have been used to produce spin-polarized electrons although these sources have used only CW lasers to date. ${ }^{25,26}$ Such magnetized sources have a further limitation in that their spin polarization is not optically reversible, unlike that of GaAs photocathodes. An array of etched GaAs tips, illuminated with CW laser light for both positive electron affinity (PEA) and NEA surface conditions, resulted in a maximum polarization of $37 \%$, but the electrons were not pulsed. ${ }^{27}$ Implementation of a tip geometry results in field enhancement at the tip apex, which increases the yield of emitted electrons. While a more robust activation surface of layered Cs and Te has been demonstrated, ${ }^{28}$ a tip geometry, as well as a multiphoton emission process, eliminates the need for NEA activation that is sensitive to vacuum conditions. ${ }^{29}$

The work reported here focuses on obtaining fast, spinpolarized electrons from a sharp $p$-GaAs bulk [110] crystal shard, which naturally incorporates optical reversibility. To do this, Ti:Sapphire pulsed lasers with wavelengths centered around $800 \mathrm{~nm}$, the appropriate wavelength for single-photon excitation across the bandgap, were used to induce multiphoton emission without requiring the samples to have NEA. Figure 1(b) illustrates this. The vacuum potential (dashed black line) is modified at the surface by the application of a negative DC bias voltage $\mathrm{V}$ and the local laser field (solid black line). A single photon with energy just exceeding the bandgap $\Delta$ can promote an electron from the valence band to the conduction band. Absorption of a second photon can in principle result in emission via tunneling through the vacuum potential (blue arrow). Absorption of one or more additional photons provides sufficient energy for the electron to exceed the additional ionization energy $\phi$ and escape into the vacuum (red arrow). The $800 \mathrm{~nm}$ central wavelength of our lasers accesses the relative excitation probabilities for circularly polarized light, which make standard NEA GaAs sources produce polarized electrons [Fig. 1(c)].

We used two apparatuses, the first to measure electron polarization and emission dichroism and the second to study the emission process duration and the emission position dependence. Our first optical setup consisted of a Ti:Sapphire oscillator (Griffin, KMLabs) with an output that passed through a collimating lens and a periscope placed prior to polarizing optics. A half-wave plate (HWP) followed by a linear polarizer was used to vary the laser power without changing the direction of its linear polarization. The beam then passed through a quarter-wave plate to switch its polarization from linear to leftor right-handed circular. A final HWP was used to rotate the plane of polarization of linearly polarized beams. The laser then entered the polarization/dichroism vacuum system through a window (Fig. 2). Just before entering the chamber, the width of the laser pulses was measured to be 75 fs using a Swamp Optics Frequency-Resolved Optical Gate (FROG).

The vacuum system, with a nominal base pressure of $10^{-7}$ Torr, is composed of two sections. A sample chamber contained an off-axis front-surface Au parabolic mirror to change the direction of the laser and focus it to a $20 \mu \mathrm{m}$-FWHM spot size. The GaAs shard was mounted on a 3-axis stage to position it in the laser focus. A channel electron multiplier (CEM) near the sample monitored the electron emission current. We also measured the total emission current from the electrically isolated sample. Emitted electrons were directed to a compact, cylindrical Mott polarimeter ${ }^{30}$ comprising two concentric cylindrical electrodes and two CEMs placed symmetrically about the entrance that defined the electron scattering plane. The central gold-plated electrode was biased at $+20 \mathrm{kV}$, whereas the outer electrode and the mouths of the CEMs were biased at $+500 \mathrm{~V}$. 


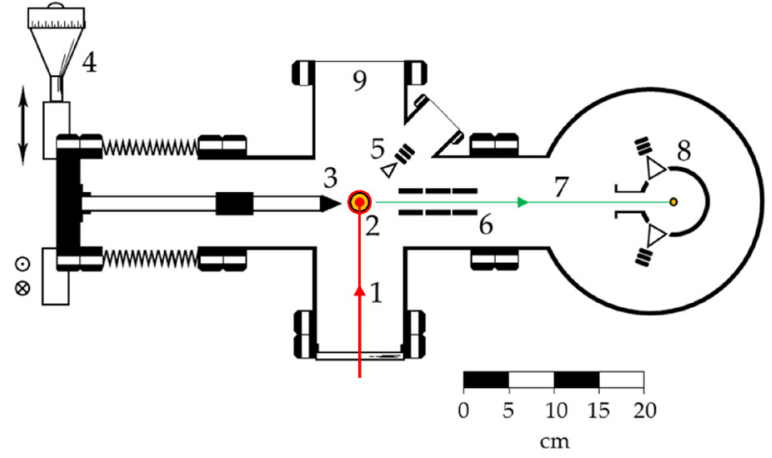

FIG. 2. The experimental setup for polarimetry and dichroism measurements. The pulsed laser beam (1) enters the chamber and hits the off-axis parabolic mirror (2) which focuses the laser onto the sample (3). Note that the beam is propagating out of the plane at (2), indicated by the red circle. The sample is mounted on an XYZ translator (4) that allows the sample tip to be positioned in the laser focus. A CEM (5) can be used to monitor electron emission. Transport optics (6) guide emitted electrons (7) toward the Mott polarimeter (8) in the adjoining chamber with top (T) and bottom (B) CEM detectors. A 260 l/s turbomolecular pump (9) evacuates the chamber.

To measure the electron polarization, $\mathrm{P}_{e}$, the count rates measured by the top and bottom CEMs $\left(C_{T}\right.$ and $\left.C_{B}\right)$ were monitored for electrons produced by light pulses that were righthand circularly polarized and then compared with the rates when the light helicity was flipped. The electron polarization is given by $\mathrm{P}_{e}=\mathrm{S}_{\mathrm{eff}} / \mathrm{A}$, where

$$
\mathrm{A}=\frac{\chi-1}{\chi+1} \quad \text { and } \quad \chi=\sqrt{\frac{\mathrm{C}_{\mathrm{T}} \mathrm{C}_{\mathrm{B}}^{\prime}}{\mathrm{C}_{\mathrm{T}}^{\prime} \mathrm{C}_{\mathrm{B}}}}
$$

Here, $S_{\text {eff }}$, the "effective Sherman function," is the polarimeter's analyzing power and the primes indicate the CEM rates for lefthanded incident laser light. The advantage of measuring $P_{e}$ this way is that it eliminates first-order instrumental asymmetries. ${ }^{17}$

Measurements of the linear and circular emission dichroism were performed to better understand the emission process. The dichroism, calculated using total emission as measured by the CEM proximate to the sample, is

$$
\mathrm{D} \equiv \frac{\mathrm{R}_{1}-\mathrm{R}_{2}}{\mathrm{R}_{1}+\mathrm{R}_{2}}
$$

where $R_{1,2}$ is the rate of emission for orthogonal polarizations.

Electron emission from the samples was optimized at the edge of the crystal shard. Sharp tip-like shards were made by shattering crystalline wafers and using an optical microscope to determine the "sharpest" pieces. ${ }^{31}$ When using these, total emission currents between $50 \mathrm{pA}$ and $3 \mathrm{nA}$ were obtained with an average laser power of $\sim 100 \mathrm{~mW}$, a pulse repetition rate of $80 \mathrm{MHz}$, and a DC sample bias of $-100 \mathrm{~V}$.

A second, similar apparatus was used to study emission rates as a function of the shard apex morphology, to measure the dependence of the emission rate on laser intensity, and to assess the temporal width of the emission process. ${ }^{32}$ Pulses from a Ti:Sapphire oscillator (Spectra Physics Tsunami) were focused to a FWHM of $3.6 \mu \mathrm{m}$. The laser pulse intensity FWHM, $\tau_{\text {laser }}$, was measured to be $100 \mathrm{fs}$. The laser power delivered to the shard apex was controlled by a Brewster window variable attenuator. Pulsed electron emission was detected using a microchannel plate (MCP) placed close to the shard apex or using an electrometer connected directly to the sample. Prior to entering the chamber, the primary beam was split into pump and probe components in a balanced Mach-Zehnder interferometer. The delay $\tau$ between pump and probe pulses could be adjusted for values between \pm 4 ps.

When two temporally separated light pulses hit the sample, the integrated electron emission can be categorized as either "additive" or "super-additive." Additive emission means that the integrated signal is the same as the sum of the emission from each pulse individually. Super-additivity occurs when the emission is greater than the sum of that due to the individual beams. Additive emission for $\tau>\tau_{0}$ shows that the emission process does not exceed $\tau_{0}$; if $\tau_{0} \approx \tau_{\text {laser }}$, the emission process is "fast" as defined in the introduction. Superadditivity for $\tau \gg \tau_{\text {laser }}$ implies that the process is slow, e.g., due to thermally assisted processes. $^{12,13,32,33}$

We first consider the electron pulse emission process. Electron emission from nanotips, if measured to be both nonlinear and additive for $\tau>\tau_{\text {laser }}$, has been shown to be fast. ${ }^{12-14}$ Our electron emission current shows non-linearity as a function of intensity. It fits with a power law of $n=5.14(16)$ [Fig. 3(a) inset; blue line]. The Keldysh parameter for a solid, $\gamma$, characterizes the emission. For $\gamma \gg 1$, field emission is dominated by multiphoton processes. $^{34,35}$ Given our focal spot sizes of $20 \mu \mathrm{m}$ and $3.6 \mu \mathrm{m}$ and an average power that never exceeded $150 \mathrm{~mW}$, our Keldysh parameter readily satisfied this condition in all our experiments and supports our simple multi-photon model. The fifth order non-linearity indicates a five-photon process. [This result is in excess of the three-photon process illustrated in Fig. 1(b)]. Generally speaking, the order of the multiphoton process in a given sample can vary with the details of the emitting surface, its local surface electric field, and the nature of surface states near the emission point. ${ }^{36}$

Pump-probe measurements as described above were used to determine if the emission was additive. ${ }^{12,32}$ The additivity ratio is defined as

$$
\mathcal{R}(\tau) \equiv \frac{\mathrm{R}_{\text {both }}(\tau)-\left(\mathrm{R}_{\text {pump }}(\tau)+\mathrm{R}_{\text {probe }}(\tau)\right)}{\mathrm{R}_{\text {pump }}(\tau)+\mathrm{R}_{\text {probe }}(\tau)},
$$

where $R_{\text {pump }}(\tau)$ and $R_{\text {probe }}(\tau)$ are the emission rates from the pump and probe beams at each delay respectively, and the rate $R_{\text {both }}(\tau)$ was modeled as

$$
R_{\text {both }}(\tau)=\int_{-\infty}^{\infty}\left[E_{\text {pump }}(t) \pm E_{\text {probe }}(t+\tau)\right]^{2 n} d t .
$$

The individual pump and probe field amplitudes were modeled as Gaussians with $E(t)=E_{0} \exp \left[-\left(t / \tau_{\text {pulse }}\right)^{2}\right]$. The best fit to the data [red line in Fig. 3(a)] is obtained for $\tau_{\text {pulse }}=160 \mathrm{fs}(n=5)$. The electron emission process is additive $[\mathcal{R}(\tau)=0$ for $\tau>400 \mathrm{fs}]$ and is thus shown to be faster than this value. Note that this is not a direct measurement of the electron pulse duration. 

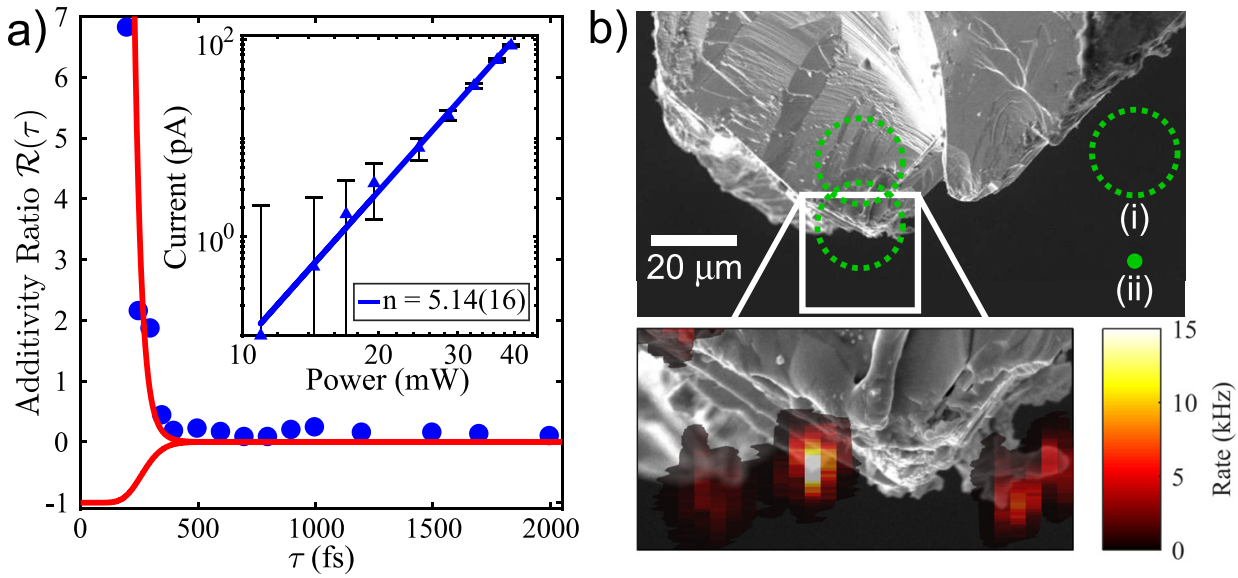

FIG. 3. Emission data from a GaAs shard "tip." In (a), $\mathcal{R}$ is plotted vs. $\tau$ (blue circles). The red line is the theoretical curve obtained with an electric field width of $160 \mathrm{fs}$ and an $p^{5}$ intensity dependence. The bifurcation of the $\mathcal{R}(\tau)$ curve for $\tau<400 \mathrm{fs}$ is due to the flipping of the sign in Eq. (4) of $E_{\text {probe }}$ and corresponds to the envelope function for the rapidly oscillating autocorrelation interference pattern in this region. The power dependence of emission is plotted in the inset. (b) A scanning electron microscopy (SEM) micrograph of the apex area with an expanded square section $20 \mu \mathrm{m}$ on a side. The laser focal spot size from the $P_{e}$ measurements [dashed green circle, (i)] is shown to the scale of the top micrograph and compared with the focal spot size [solid green circle, (ii)] used for the measurements shown in (a). The tip and shank positions used for measurements of $P_{e}$ and $D$ are indicated by the $20 \mu \mathrm{m}$ dashed green circles (bottom and top, respectively). Localized emission from the shard's sharpest features (inset) indicates that multiple sites may have been emitting in the $P_{e}$ and $D$ measurements.

Nevertheless, fast emission processes have so far indicated short electron pulses. ${ }^{35}$ For emission rates with an average of less than one electron per pulse, this is not surprising, given that space charge effects are essentially absent.

We now turn our attention to electron polarization. Measurements of $\mathrm{P}_{e}$ were taken with a $20 \mu \mathrm{m}$-diameter focal spot for two focal positions on the three samples we studied. In the first "tip" position, the focal spot was centered on the shard apex. In the second "shank" position, the focus center was moved about $15 \mu \mathrm{m}$ away from the tip towards the bulk. The results of all measurements of $\mathrm{P}_{e}$ and emission dichroism, taken with the $20 \mu \mathrm{m}$ focus, are given in Table I. In the "tip" position, with circularly polarized laser illumination, $\mathrm{P}_{e}$ was $13 \%$ for samples 1 and 2 and 10\% for sample 3 . Note that these results are comparable to those of Ref. 24. Variations in the local structure or $p$-doping could be responsible for the differences in $\mathrm{P}_{e}{ }^{36}$ Each $P_{e}$ value is the result of between 4 and 87 runs, typically

TABLE I. Polarization and dichroism results for circularly and linearly polarized light incident on either the apex ("tip") or the bulk ("shank") of three different shard samples.

\begin{tabular}{lccc}
\hline \hline Target & Light Polarization & $P_{e}(\%)$ & $\mathrm{D}(\%)$ \\
\hline \#1 Tip & Circular & $13.1(0.9)$ & \\
\#2 Tip & Circular & $13.3(0.7)$ & $4.7(0.6)$ \\
& Linear & $0.1(0.5)$ & $41.3(1.0)$ \\
\#3 Tip & Circular & $10.4(0.2)$ & $1.8(0.2)$ \\
& Linear & $2.6(2.5)$ & $18.5(0.6)$ \\
\#1 Shank & Circular & $1.7(8.0)$ & $6.4(1.4)$ \\
& Linear & $1.0(2.1)$ & $23.7(5)$ \\
\#2 Shank & Circular & $3.4(1.6)$ & \\
& Linear & $5.2(1.0)$ & \\
\hline \hline
\end{tabular}

taken over several hours. Individual runs yielded uncertainties that we based on counting statistics alone. Occasionally, these error bars were increased to account for small temporal drifts. The values quoted in Table I are the uncertainty-weighted averages of these individual run values. As expected, when the laser was linearly polarized, the values of $\mathrm{P}_{e}$ were consistent with zero. One exception, which we have yet to understand, was observed with sample 2 in the shank position. We note only that this value of $\mathrm{P}_{e}$ is less than half that of the polarization measured at the tip with circularly polarized light.

Finally, we consider the sample morphology. The electron emission rate was found to depend sensitively on the position of the laser focus at the sample. Figure 3(b) shows a plot of the emission rate measured in a $20 \mu \mathrm{m}$ square area of a shard apex. The two laser focal spot sizes used in this work are shown relative to the size of the $20 \mu \mathrm{m}$ scale bar in the top micrograph. The brightest emission feature was used for the measurements plotted in Fig. 3(a).

Non-zero linear emission dichroism [Eq. (2)] was observed for the GaAs shards similar to a field emission tip (FET). That is, emission is higher when the light's linear polarization is parallel to the axis of the tip. ${ }^{12,13}$ In contrast, emission dichroism is absent for standard planar GaAs sources. ${ }^{16,17}$ Dichroism measurements were taken at both focal positions as well. At the tip of GaAs, the circular dichroism is small $(<5 \%)$ and the linear dichroism for tips 1 and 2 is $41 \%$ and $19 \%$, respectively. Linear dichroism measured for tip 1 drops to $24 \%$ at the shank, possibly because there is less of a tip-like structure with which the light interacts. Thus, our shard "tips" have emission characteristics similar to those of FETs ${ }^{12-15}$ in terms of nonlinearity, additivity, polarization, and local morphology, although it is apparent from Fig. 3(b) that the overall shard morphology is complex. 
In summary, we have demonstrated a source that is able to produce fast pulses of polarized electrons from a micrometersize area. This can, in principle, enable the imaging of a small electron spot on a target to measure spin-dependent effects with fs-scale resolution. The reduced vacuum requirements of this source when compared with NEA GaAs sources make it easier and less costly to operate. Although the observed electron polarization is modest, our results demonstrate that this source follows the selection rules illustrated in Fig. 1(c). Polarization might be increased by having a sharper, more well-defined GaAs tip or varying the laser wavelength. The parameter space is large and open to future study. Through the use of chemical etching and ion milling, it is possible to shape the tip. An optical parametric amplifier can be used to explore the wavelengthdependence of polarization. Investigation of the effects these parameters have on the total yield and polarization of the emitted electrons is needed.

We thank M. Becker for useful conversations and S. Keramati for taking the electron micrographs shown in Fig. 3(b). This work was funded by NSF Award Nos. PHY-1206067 and 1505794 (T.J.G.), EPS-1430519 (H.B. and T.J.G.), and PHY1602755 (H.B.).

\section{REFERENCES}

${ }^{1}$ V. A. Lobastov, R. Srinivasan, and A. H. Zewail, Proc. Natl. Acad. Sci. U. S. A. 102, 7069 (2005).

${ }^{2}$ P. Lougovski and H. Batelaan, Phys. Rev. A 84, 023417 (2011).

${ }^{3}$ E. Jones, M. Becker, J. Luiten, and H. Batelaan, Laser Photonic Rev. 10, 214 (2016).

${ }^{4} \mathrm{C}$. Ropers and M. Kasevich, personal communications (November 30, 2018).

${ }^{5}$ H. Kiesel, A. Renz, and F. Hasselbach, Nature 418, 392 (2002).

${ }^{6}$ T. Kodama, N. Osakabe, and A. Tonomura, Phys. Rev. A 83, 063616 (2011).

${ }^{7} \mathrm{G}$. Baym and K. Shen, "Hanbury Brown-Twiss interferometry with electrons: Coulomb vs. quantum statistics," in In Memory of Akira Tonomura (World Scientific, 2014), pp. 201-210.

${ }^{8}$ A. H. Zewail, Science 328, 187 (2010).

${ }^{9}$ R. Bormann, S. Strauch, S. Schäfer, and C. Ropers, J. Appl. Phys. 118, 173105 (2015).

${ }^{10}$ M. T. Hassan, J. S. Baskin, B. Liao, and A. H. Zewail, Nat. Photonics 11, 425 (2017).

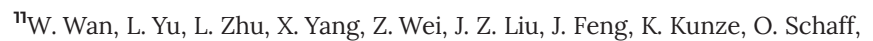
R. Tromp, and W.-X. Tang, Ultramicroscopy 174, 89 (2017).

${ }^{12}$ B. Barwick, C. Corder, J. Strohaber, N. Chandler-Smith, C. Uiterwaal, and H. Batelaan, New J. Phys. 9, 142 (2007).

${ }^{13}$ P. Hommelhoff, Y. Sortais, A. Aghajani-Talesh, and M. A. Kasevich, Phys. Rev. Lett. 96, 077401 (2006).

${ }^{14}$ P. Hommelhoff, C. Kealhofer, and M. A. Kasevich, Phys. Rev. Lett. 97, 247402 (2006).

${ }^{15}$ C. Ropers, D. R. Solli, C. P. Schulz, C. Lienau, and T. Elsaesser, Phys. Rev. Lett. 98, 043907 (2007).

${ }^{16}$ D. T. Pierce and F. Meier, Phys. Rev. B 13, 5484 (1976).

${ }^{17}$ T. J. Gay, Adv. At. Mol. Phys. 57, 157 (2009).

${ }^{18}$ D. Androic, D. S. Armstrong, A. Asaturyan, T. Averett, J. Balewski, J. Beaufait, R. S. Beminiwattha, J. Benesch, F. Benmokhtar, J. Birchall et al., Phys. Rev. Lett. 111, 141803 (2013).

${ }^{19}$ F. Giebels, H. Gollisch, and R. Feder, Phys. Rev. B 87, 035124 (2013).

${ }^{20}$ K. Aulenbacher, J. Schuler, D. v Harrach, E. Reichert, J. Röthgen, A. Subashev, V. Tioukine, and Y. Yashin, J. Appl. Phys. 92, 7536 (2002).

${ }^{21}$ M. Kuwahara, S. Kusunoki, X. G. Jin, T. Nakanishi, Y. Takeda, K. Saitoh, T. Ujihara, H. Asano, and N. Tanaka, Appl. Phys. Lett. 101, 033102 (2012).

${ }^{22}$ M. Kuwahara, S. Kusunoki, Y. Nambo, K. Saitoh, X. Jin, T. Ujihara, H. Asano, Y. Takeda, and N. Tanaka, Appl. Phys. Lett. 105, 193101 (2014).

${ }^{23}$ M. Kuwahara, Y. Nambo, K. Aoki, K. Sameshima, X. Jin, T. Ujihara, H. Asano, K. Saitoh, Y. Takeda, and N. Tanaka, Appl. Phys. Lett. 109, 013108 (2016).

${ }^{24}$ P. Klaer, F. Schertz, M. Lehr, G. Schönhense, and H. J. Elmers, Phys. Rev. B 88, 214425 (2013).

${ }^{25}$ T. Irisawa, T. K. Yamada, and T. Mizoguchi, New J. Phys. 11, 113031 (2009).

${ }^{26}$ Y. Niu and M. Altman, Surf. Sci. 604, 1055 (2010).

${ }^{27}$ M. Kuwahara, T. Nakanishi, S. Okumi, M. Yamamoto, M. Miyamoto, N. Yamamoto, K. Yasui, T. Morino, R. Sakai, K. Tamagaki, and K. Yamaguchi, Jpn. J. Appl. Phys., Part 145, 6245 (2006).

${ }^{28}$ J. K. Bae, L. Cultrera, P. DiGiacomo, and I. Bazarov, Appl. Phys. Lett. 112, 154101 (2018).

${ }^{29}$ M. Pirbhai, J. Knepper, E. T. Litaker, D. Tupa, and T. J. Gay, Phys. Rev. A 88, 060701 (2013).

${ }^{30}$ N. B. Clayburn, E. Brunkow, S. J. Burtwistle, G. H. Rutherford, and T. J. Gay, Rev. Sci. Instrum. 87, 053302 (2016).

${ }^{31}$ M. W. J. Prins, R. Jansen, and H. van Kempen, Phys. Rev. B 53, 8105 (1996).

${ }^{32}$ E. R. Jones, W. C. Huang, G. Basnet, B. N. Flanders, and H. Batelaan, Appl. Phys. Lett. 112, 263104 (2018).

${ }^{33}$ C. Kealhofer, S. M. Foreman, S. Gerlich, and M. A. Kasevich, Phys. Rev. B 86, 035405 (2012).

${ }^{34}$ F. V. Bunkin and M. V. Federov, Sov. Phys. JETP 5, 896 (1965).

${ }^{35}$ M. Krüger, M. Schenk, M. Förster, and P. Hommelhoff, J. Phys. B 45, 074006 (2012).

${ }^{36}$ O. Schmidt, M. Bauer, C. Wiemann, R. Porath, M. Scharte, O. Andreyev, G. Schönhense, and M. Aeschlimann, Appl. Phys. B 74, 223 (2002). 